\title{
A mechanism of orbital reconstruction at the interfaces of transition metal oxides
}

\author{
Natalia Pavlenko \\ Institute for Condensed Matter Physics, 79011 Lviv, Ukraine \\ EKM, Universität Augsburg, 86135 Augsburg, Germany
}

(Dated: December 3, 2018)

\begin{abstract}
Orbital reconstruction at interfaces between $\mathrm{YBa}_{2} \mathrm{Cu}_{3} \mathrm{O}_{6}$ and $\mathrm{SrO}$-terminated $\mathrm{SrTiO}_{3}$ is studied using local spin density approximation (LSDA) with intra-atomic Coulomb repulsion (LSDA $+U$ ). The change of population of interfacial $\mathrm{Cu} 3 d$ orbitals results in stabilization of a new oxidation state $3 d^{8}$ which involves an additional modification of orbital occupancies in the nearest $\mathrm{SrO}$ and $\mathrm{TiO}_{2}$ layers. We find that an increase of electron charge in $\mathrm{Cu} 3 d_{x^{2}-y^{2}}$ states counterbalances a depopulation of $3 d_{3 z^{2}-r^{2}}$ orbitals which induces, on account of the onsite Coulomb repulsion $U$, a splitting of $3 d_{3 z^{2}-r^{2}}$ states at $\mathrm{CuO}_{2}-\mathrm{SrO}$ interfaces.

PACS numbers: 74.78.Fk,74.78.-w,73.20.-r
\end{abstract}

\section{INTRODUCTION}

Interfaces between transition metal oxides attract much attention due to their technological and fundamental importance. In a complex transition metal oxide system, new electronic states at the interfaces between different components can bring dramatic changes into its physical properties. The prominent examples are metallic mixed-valence heterostructures of $\mathrm{SrTiO}_{3}$ and $\mathrm{LaTiO}_{3}$ and the quasi-two-dimensional electron gas at the interface between two insulators $\mathrm{SrTiO}_{3}$ and $\mathrm{LaAlO}_{3}$ with recently discussed superconducting properties ${ }^{1,2,3}$.

In the transition metal oxide heterostructures, the new interfacial electronic states can be driven by the mechanisms of electronic reconstructions ${ }^{4}$. These mechanisms involve charge compensation of interface polarity or charge self-doping due to interface chemical abruptness which is one of the ways to maintain the overall electrostatical neutrality of the system ${ }^{5}$. Besides the well understood scenarios of interface charge doping, other mechanisms of interface reconstructions can play a decisive role in the unusual electronic properties of transition metal systems. Recently the so called orbital reconstruction has been shown to occur at the interfaces between cuprate and manganite films which results in changes of $3 d$ orbital occupancies of the interfacial copper ions 6 .

The first evidence for the interface orbital reconstruction came from resonant $\mathrm{x}$-ray spectroscopy studies of $\mathrm{YBa}_{2} \mathrm{Cu}_{3} \mathrm{O}_{7} / \mathrm{LaCaMnO}_{3}$-heterostructures 6 . In these experiments, the x-ray spectral intensity at the interface between $\mathrm{YBa}_{2} \mathrm{Cu}_{3} \mathrm{O}_{7}$ and $\mathrm{La}_{0.67} \mathrm{Ca}_{0.33} \mathrm{MnO}_{3}$ is found to be similar for the photon polarization in $(x, y)$-plane and in $z$ direction, which is interpreted in terms of the appearance of hole charge in $3 d_{3 z^{2}-r^{2}}$ orbitals. This is in strong contrast to the standard hole doping mechanism where the holes occupy $\mathrm{O} 2 p$ orbitals of the Zhang-Rice singlets in $\mathrm{Cu}^{+2} \mathrm{O}_{2}^{-4}$ planes of $\mathrm{YBa}_{2} \mathrm{Cu}_{3} \mathrm{O}_{7-\delta}$. The $3 d_{3 z^{2}-r^{2}}$ orbital reconstruction has been explained by a formation of a new "extended molecular orbital" at the oxide interfaces in which a substantial amount of hole charge is distributed over the $\mathrm{Mn}, \mathrm{Cu}$ and $\mathrm{O}$ orbitals. As is shown in Refs. 6. 8, the high Mn charge +3 results in an electron transfer from $\mathrm{Mn}$ to $3 d_{x^{2}-y^{2}}$ orbitals of $\mathrm{Cu}$, whereas the $\mathrm{Cu} 3 d_{3 z^{2}-r^{2}}$ states become partially occupied. Moreover, in such an electron doped system, the coexistence of two partially occupied $e_{g}$ orbitals leads to the formation of Zhang-Rice triplets centered on $\mathrm{Cu}$ with the total spin $S=1$. In contrast, for the hole-doped interfaces, the author in Ref. 8 expects a rather standard $x^{2}-y^{2}$-singlet character of the electron configuration with a stabilization of triplet states when the hole density is increased. As is shown in Ref. 8, the singlet character of the electron state can be possibly changed due to structural JahnTeller distortions.

In the present work, we explore theoretically a mechanism of the orbital reconstruction occurring through the splitting of $3 d_{3 z^{2}-r^{2} \uparrow \uparrow}$ and $3 d_{3 z^{2}-r^{2}, \downarrow}$ bands by the interface charge redistribution. As an example of a system where the interface-caused splitting of $3 d_{3 z^{2}-r^{2}}$ can be obtained within the density functional theory, we consider a supercell with a $\mathrm{CuO}_{2}$ layer directly deposited on the top of a $\mathrm{SrTiO}_{3}$ slab terminated by $\mathrm{SrO}$, a model oxide interface recently studied in Refs. 9.10 and shown schematically in Fig. 1. In this model system, the electrostatic polarity of the interfaces between $\mathrm{CuO}_{2}$ and $\mathrm{SrO}$ could be compensated via the interface self-doping by holes. Like in the bulk $\mathrm{YBa}_{2} \mathrm{Cu}_{3} \mathrm{O}_{7-\delta}$, in heterostructures the interface self-doped holes in the $\mathrm{CuO}_{2}$ layers are accumulated predominantly in the $\mathrm{O} 2 p$ orbitals. Although the charge self-doping mechanism appears to be a common scenario in a wide variety of polar oxide interfaces, a close examination of the electronic structure in the $\mathrm{CuO}_{2} / \mathrm{SrO}$ region unexpectedly shows that a mechanism different from the electrostatic self-doping plays a key role ${ }^{10}$.

\section{INTERFACE CRYSTAL FIELDS AND STRUCTURAL RELAXATION}

As appears from the earlier $\mathrm{LDA}+U$ studies $^{9,10}$, the relaxation of the interfacial distance $h$ between $\mathrm{CuO}_{2}$ and $\mathrm{SrO}$ planes results in the optimized value $h=h_{0}=$ $1.83 \AA$ which is by $0.5 \AA$ smaller than the distance be- 


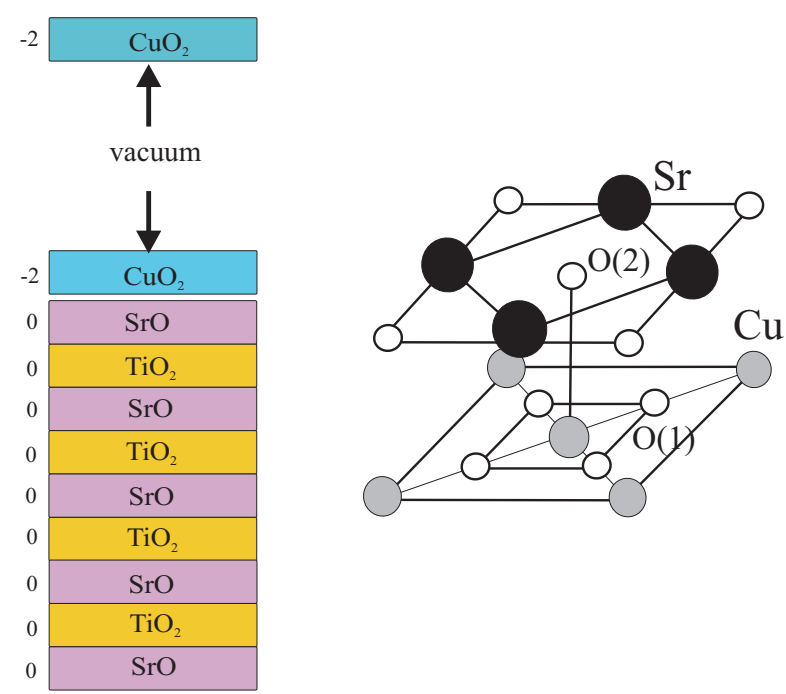

FIG. 1: Scheme of a polar $\mathrm{CuO}_{2} / \mathrm{SrTiO}_{3}$-superlattice where a STO-layer is terminated by a SrO-plane. The right side shows a structural configuration which appears at the interface.

tween the apical oxygen $\mathrm{O}(2)$ and $\mathrm{Cu}$ in $\mathrm{CuO}_{2}$ planes of the bulk $\mathrm{YBa}_{2} \mathrm{Cu}_{3} \mathrm{O}_{6}$. For larger interface distances $h \geq 2.2 \AA$, the compensation of the polarity proceeds through a standard self-doping mechanism of the electronic reconstruction and leads to an extremely high selfdoping level $n_{h}=+2$ holes/per unit cell which enforces charge neutrality (cf. the formal valency of each layer in Fig. 1 to the left of the block scheme). As can be seen in Fig. 2, the holes are located in $\mathrm{O}(1) 2 p_{x, y}$ orbitals (top panel, dashed arrows), in close analogy to the Zhang-Rice singlets described by a $3 d^{9}$ configuration in the $\mathrm{CuO}_{2}$ planes.

In contrast to the unrelaxed interfaces, for the optimized distances $h \sim h_{0}$ the new (orbital) mechanism of the compensation of the interface polarity becomes equally important. This new mechanism is associated with a change of the occupancy of one of the $3 d_{3 z^{2}-r^{2}}$ orbitals from one to zero due to a "displacement" of this band above the Fermi level (see Fig. 31). From the point of view of classical chemical valence theory, the resulting electronic configuration can be formally described as a " $3 d^{8}$ " state. In Fig. 2, the charge and orbital reconstructions caused by the $+2 e$-doping level are schematically shown for comparison. Formally, a $\mathrm{Cu} 3 d^{8}$ configuration corresponds to a change of the valency of $\mathrm{Cu}$ from +2 to +3 and is typically considered as unstable in the literature. The expectation of such an instability was the reason for a preference for the standard self-doping reconstruction mechanism in our earlier discussion of the $\mathrm{CuO}_{2} / \mathrm{SrTiO}_{3}$ system in Ref. 9. However, a further close and careful examination of the electronic reconstruction in this system shows a more complex scenario which is beyond a simple integer-like change of the electron charge on the copper ion (Fig. 2 bottom panel). In this mechanism, the modification of the oxidation state of $\mathrm{Cu}$ can

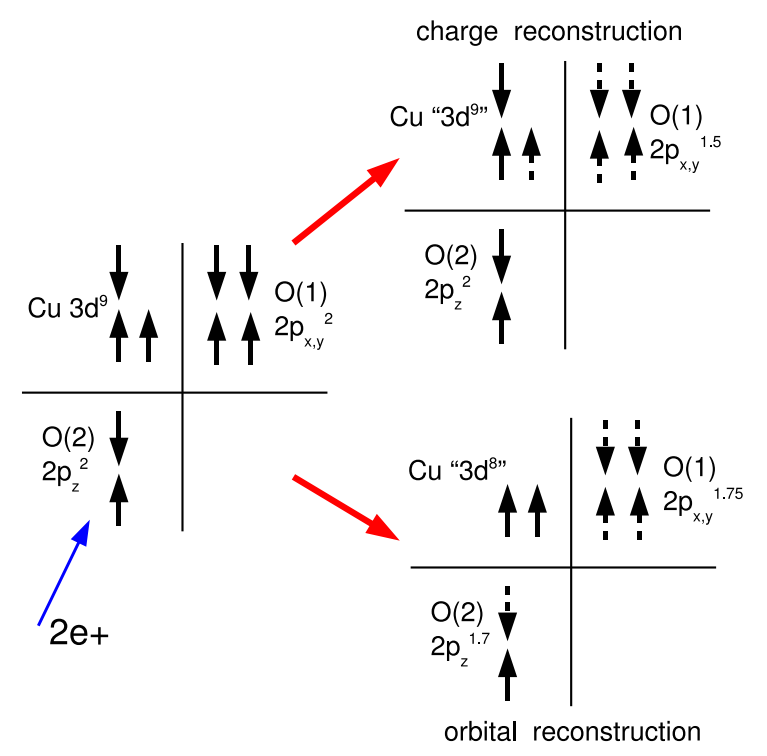

FIG. 2: Schematic view of the electron configurations which result from hole doping. The top and bottom right panels show the electronic and orbital reconstructed configurations with black arrows denoting the electronically fully occupied (bold arrows) and partially occupied (dashed arrows) orbitals. The configurations " $3 d^{8} "$ and " $3 d^{9}$ " formally describe the states $3 d^{8}$ and $3 d^{9}$ with partially occupied $e_{g}$ orbitals.

be described by two steps, namely (i) by a depletion of electron charge from a $3 d_{3 z^{2}-r^{2}}$ band and from the $2 p_{z}$ band of $\mathrm{O}(2)(\mathrm{SrO})$, and (ii) by an increase of electron density in the $3 d_{x^{2}-y^{2}}$ orbitals hybridized with $\mathrm{O}(1) 2 p_{x, y}$ states. These two steps provide jointly a decrease of the net electron charge on $\mathrm{Cu}$ which is an additional way to compensate the interface polarity. Consequently, in the relaxed structure the electrostatic neutrality is achieved by a combination (a) of the standard self-doping mechanism with the self-doped hole charge $n_{h}=1$ on $\mathrm{O} 2 p$ orbitals (Fig. 2, top panel), and (b) of the $3 d_{3 z^{2}-r^{2}}$ orbital reconstruction which leads to a decrease of the local electron charge on $\mathrm{Cu}$ (Fig. 2, bottom panel).

Let us estimate the crystal field which would be sufficient for stabilization of the new oxidation state formally denoted here as extended $\mathrm{Cu} 3 d^{8}$. Using the notations $\{\uparrow, \downarrow\}$ for the majority and minority spin states, the $3 d^{8}$ state can be described as $3 d^{5 \uparrow, 3 \downarrow}$ configuration where the occupied states $3 d_{x^{2}-y^{2}, \uparrow}$ and $3 d_{3 z^{2}-r^{2}, \uparrow}$ have a character of a Zhang-Rice triplet, similar to the results of Ref. 8 . In the relaxed structure, this configuration corresponds to the minimum of the total energy. In the LDA $+U$ calculations, the energy gain $\Delta E_{t o t}=E_{t o t}\left(h_{1}\right)-E_{t o t}\left(h_{0}\right)$ due to the relaxation of the interface distance from $h=h_{1}=2.2 \AA$ to $h=h_{0}$ approaches $1 \mathrm{eV}$. It is remarkable that in the extended $\mathrm{Cu} 3 d^{8}$ configuration, the main contribution to $\Delta E_{t o t}$ is provided by the reconstruction of the electronic states in the nearest $\mathrm{CuO}_{2}$ and $\mathrm{SrO}$ 


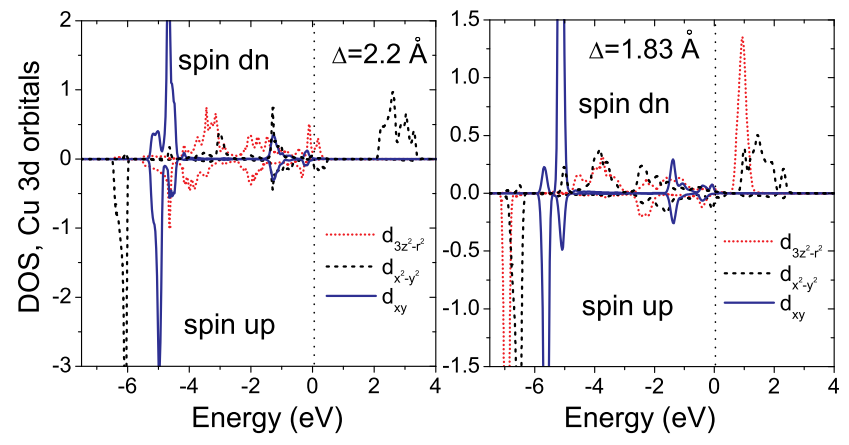

FIG. 3: Partial $\mathrm{Cu} 3 d$ orbital density of states at the interface between $\mathrm{CuO}_{2}$ and $\mathrm{SrTiO}_{3}$ terminated by $\mathrm{SrO}$ (LSDA $+U$ studies). The zero of energy is at the Fermi level. The left and right panels correspond to the case of unrelaxed $(h=2.2 \AA)$ and optimized ( $h=1.83 \AA$ ) interfacial distances, respectively.

planes

$$
\begin{aligned}
\Delta E_{t o t}= & \Delta \varepsilon_{3 z^{2}-r^{2}}+\Delta \varepsilon_{x^{2}-y^{2}} \\
& +\Delta \varepsilon_{x z+y z}+\Delta p_{O}^{2},
\end{aligned}
$$

where $\Delta \varepsilon_{3 z^{2}-r^{2}}, \Delta \varepsilon_{x^{2}-y^{2}}$, and $\Delta \varepsilon_{x z+y z}$ are the changes of the $3 d$ orbital energies; $\Delta p_{O}^{2}$ refers to the shifts of the energies of the $2 p$ bands of oxygens $\mathrm{O}(2)$. In our calculations, the band energies are represented by their gravity centers $\frac{11.12}{12}$. Here a gravity center $E_{g}$ of a band with a density of states $\rho(E)$ is calculated from the expression $E_{g}=\int d E E \rho(E) / \int d E \rho(E)$. As the displacements of the gravity centers of the $2 p$ states of $\mathrm{O}(1), 2 p_{x}$ and $2 p_{y}$ states of $\mathrm{O}(2)$ and $3 d_{x y}$ bands of $\mathrm{Cu}$ are negligibly small as compared to the splitting of $e_{g}, 3 d_{x z}$ and $3 d_{y z}$ bands, they are not included into (1).

In the SIC LDA $+U$ approach ${ }^{14.15}$, the $3 d_{3 z^{2}-r^{2}}$ orbital energies in the optimized $(\nu=0)$ and unrelaxed $(\nu=1)$ system can be represented as

$$
\varepsilon_{3 z^{2}-r^{2}, \sigma}^{\nu}=\varepsilon_{3 z^{2}-r^{2}}^{\nu(0)}+U_{e f f}\left(\frac{1}{2}-n_{3 z^{2}-r^{2}, \sigma}^{\nu}\right) .
$$

Here $U_{\text {eff }}=U-J$ and $\sigma$ is the spin index. In our calculations, we consider the local Coulomb corrections for $\mathrm{Cu} 3 d$ orbitals $U=8 \mathrm{eV}$, and the Hund coupling $J=0.8 \mathrm{eV}$. In the unrelaxed structure, the states $d_{3 z^{2}-r^{2}}$ are filled by electrons: $n_{3 z^{2}-r^{2}, \sigma}^{1} \approx 1$. In contrast, in the optimized system, the orbital reconstruction leads to $n_{3 z^{2}-r^{2}, \uparrow}^{0} \approx 1$ and $n_{3 z^{2}-r^{2}, \downarrow}^{0} \approx 0$. The quantities $\varepsilon_{3 z^{2}-r^{2}}^{\nu(0)}$ are the corresponding LDA orbital potentials which are assumed to be independent of the spin direction. As a consequence, the total $3 d_{3 z^{2}-r^{2}}$ energy shift for the relaxed structure is given by

$$
\begin{aligned}
& \Delta \varepsilon_{3 z^{2}-r^{2}}=\Delta \varepsilon_{3 z^{2}-r^{2}, \uparrow} n_{3 z^{2}-r^{2}, \uparrow}+\varepsilon_{3 z^{2}-r^{2}, \downarrow}^{1} n_{3 z^{2}-r^{2}, \downarrow}^{1} \\
& \approx \Delta \varepsilon_{3 z^{2}-r^{2}}^{10}+n_{3 z^{2}-r^{2}, \downarrow}^{1} \varepsilon_{3 z^{2}-r^{2}, \downarrow}^{1(0)}-\frac{1}{2} U_{e f f}
\end{aligned}
$$

where the potential difference $\Delta \varepsilon_{3 z^{2}-r^{2}}^{10}=\varepsilon_{3 z^{2}-r^{2}}^{1(0)}$
TABLE I: Band centers of gravity (in eV) for different values of interfacial distance $h$. Here, $\varepsilon_{2, z}$ refers to the $2 p_{z}$ orbital of $\mathrm{O}(2)$.

\begin{tabular}{llll}
\hline \hline$h(\AA), \sigma$ & $\varepsilon_{x^{2}-y^{2}}$ & $\varepsilon_{x z+y z}$ & $\varepsilon_{2, z}$ \\
\hline $1.83(\uparrow)$ & -5.03 & -5.23 & -2.98 \\
$1.83(\downarrow)$ & -1.07 & -4.84 & -2.62 \\
$2.20(\uparrow)$ & -4.68 & -3.60 & -1.93 \\
$2.20(\downarrow)$ & 0.97 & -3.16 & -1.82 \\
\hline \hline
\end{tabular}

$\varepsilon_{3 z^{2}-r^{2}}^{0(0)}$ is determined by the change of the orbital crystal field due to the interfacial relaxation.

From (21), the change of the splitting of the spin bands $\varepsilon_{3 z^{2}-r^{2}, \uparrow}$ and $\varepsilon_{3 z^{2}-r^{2}, \downarrow}$ due to structural relaxation can be described as

$$
\begin{aligned}
& \Delta \varepsilon_{3 z^{2}-r^{2}, \uparrow}-\Delta \varepsilon_{3 z^{2}-r^{2}, \downarrow} \\
& \quad=U_{e f f}\left(n_{3 z^{2}-r^{2}, \downarrow}^{1}-n_{3 z^{2}-r^{2}, \downarrow}^{0}\right) \approx U_{e f f} .
\end{aligned}
$$

where $n_{3 z^{2}-r^{2}, \downarrow}^{\nu}$ is the occupation of the $\left(3 z^{2}-r^{2}, \downarrow\right)$ orbital in the optimized $(\nu=0)$ and unrelaxed $(\nu=$ 1) system. The last result demonstrates that the onsite Coulomb repulsion controls the spin band splitting and the consequent enhancement of the local magnetic moments.

As the width of the $3 d_{3 z^{2}-r^{2}}$ bands exceeds the shift $\Delta \varepsilon_{3 z^{2}-r^{2}}$ caused by the orbital reconstruction, the use of the centers of gravity to estimate the changes of the crystal fields cannot be sufficiently accurate to convey the physical picture. Therefore, we consider a different analysis of these fields. In this approach, we calculate the centers of gravity of the $3 d_{x^{2}-y^{2}}$ and $t_{2 g}$ bands which are expected to be accurate due to their substantial shifts by about $2 \mathrm{eV}$ caused by the crystal field. The obtained values for $\Delta \varepsilon_{x^{2}-y^{2}}$ and $\Delta \varepsilon_{x z+y z}$ together with the similarly calculated $\Delta p_{O}^{2}$ can be directly substituted into the equation (1). For the known $\Delta E_{\text {tot }}$ this equation provides an estimate for the minimal energy $\Delta \varepsilon_{3 z^{2}-r^{2}}$

$$
\begin{gathered}
\Delta \varepsilon_{3 z^{2}-r^{2}}^{10} \approx \Delta E_{t o t}+\frac{1}{2} U_{e f f}-\Delta \varepsilon_{x^{2}-y^{2}} \\
-\varepsilon_{3 z^{2}-r^{2}}^{1(0)} n_{3 z^{2}-r^{2}, \downarrow}^{1}-\Delta \varepsilon_{x z+y z}-\Delta p_{O}^{2},
\end{gathered}
$$

which is sufficient for the stabilization of the orbitally reconstructed extended $\mathrm{Cu} 3 d^{8}$ configuration. Here $\varepsilon_{3 z^{2}-r^{2}}^{1(0)}=-1.86 \mathrm{eV}$ is the LDA-energy level of the unrelaxed $3 d_{3 z^{2}-r^{2}}$ orbital.

In expression (5), the energies of the $3 d_{x^{2}-y^{2}}$ bands can be represented as

$$
\varepsilon_{x^{2}-y^{2}, \sigma}^{\nu}=\varepsilon_{x^{2}-y^{2}, \sigma}^{\nu(0)}+U_{e f f}\left(\frac{1}{2}-n_{x^{2}-y^{2}, \sigma}^{\nu}\right) .
$$

In distinction to the reconstructed $3 d_{3 z^{2}-r^{2}}$ orbitals, the occupation numbers $n_{x^{2}-y^{2}, \sigma}^{\nu}$ of $3 d_{x^{2}-y^{2}}$ states are affected only slightly by the decrease of $h$. In (6), the 

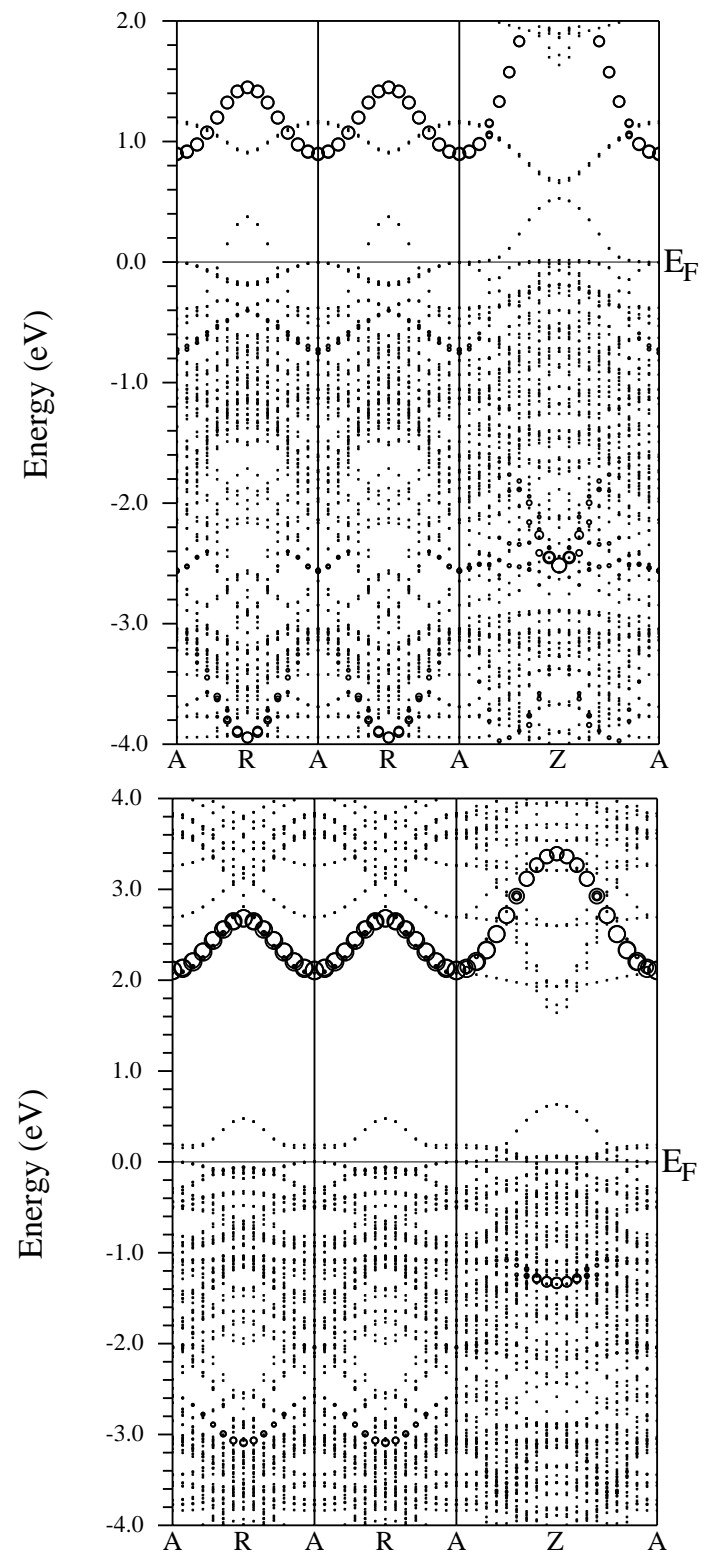

FIG. 4: Band structure for different $h$ where the top panel corresponds to $h=1.83 \AA$ and the bottom panel to $h=2.2 \AA$. The size of the circles is proportional to the $\mathrm{Cu} 3 d_{x^{2}-y^{2}, \downarrow}$ character of the respective states. Here the coordinates of the symmetric points of the Brillouin zone are: $\mathrm{A}=(-\pi, \pi)$, $\mathrm{R}=(-\pi, 0)$, and $\mathrm{Z}=(0,0)$.

latter property leads to small changes by $0.07 \mathrm{eV}$ in the second local Coulomb contribution due to atomic relaxation. Consequently, the effect of the interface relaxation on $3 d_{x^{2}-y^{2}}$ is reduced to a modification of the LDA potentials $\varepsilon_{x^{2}-y^{2}, \sigma}^{\nu(0)}$ :

$$
\Delta \varepsilon_{x^{2}-y^{2}}=\Delta \varepsilon_{x^{2}-y^{2}, \uparrow}^{(10)}+\Delta \varepsilon_{x^{2}-y^{2}, \downarrow}^{(10)}
$$

where $\Delta \varepsilon_{x^{2}-y^{2}, \sigma}^{(10)}=\varepsilon_{x^{2}-y^{2}, \sigma}^{1(0)} n_{x^{2}-y^{2}, \sigma}^{1}-\varepsilon_{x^{2}-y^{2}, \sigma}^{0(0)} n_{x^{2}-y^{2}, \sigma}^{0}$. Using the results of the $\mathrm{LDA}+U$ calculations, the shift
$\Delta \varepsilon_{x^{2}-y^{2}, \sigma}^{(10)}$ and the concentrations $n_{x^{2}-y^{2}, \sigma}^{\nu}$ can be found from the centers of gravity of $3 d_{x^{2}-y^{2}}$ bands and by integration of the densities of states obtained for the relaxed and optimized interfaces. Similarly to $\Delta \varepsilon_{x^{2}-y^{2}}$, the shift $\Delta \varepsilon_{x z+y z}$ can be easily determined from the changes of the LDA potentials $\varepsilon_{x z+y z, \sigma}^{\nu(0)}$.

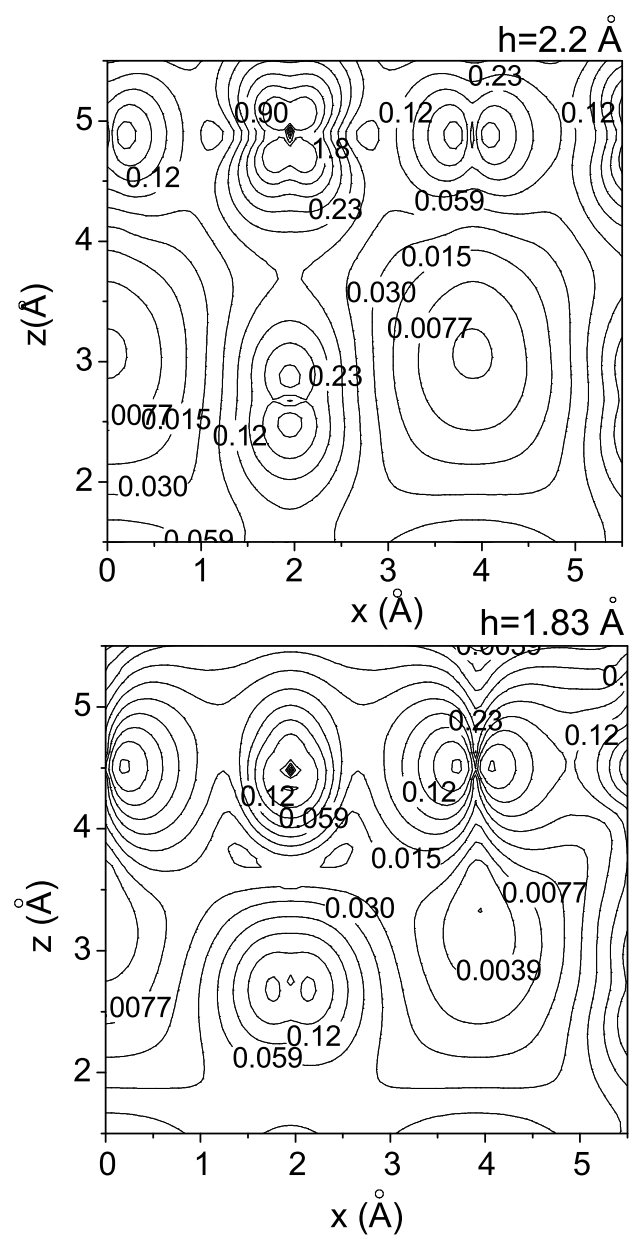

FIG. 5: Electron density map (in e/ $\AA^{3}$ ) in the energy range $-4.5 \mathrm{eV} \leq E \leq-2.5 \mathrm{eV}$ below the Fermi level for unrelaxed and optimized interface distances. Here the electron density is plotted in the $(x, z)$ plane with the $x$-axis along the $\mathrm{O}(1)$ $\mathrm{Cu}-\mathrm{O}(1)$ diagonal of the $\mathrm{CuO}_{4}$ plaquettes shown in the right panel of Fig. 1

The band gravity centers calculated for $\mathrm{Cu} 3 d_{x^{2}-y^{2}}$, $3 d_{x z+y z}$ and for $2 p_{z}$ orbitals of $\mathrm{O}(2)$ in superlattices with unrelaxed and optimized $h$ are presented in Table【. From this table, one can detect that a central feature of the interface relaxation is a strong shift of the center of the minority band $3 d_{x^{2}-y^{2}, \downarrow}$ down to the energy $-1.07 \mathrm{eV}$ below the Fermi level. The reason for this shift can be understood from the analysis of the band structures of the optimized and unrelaxed interfaces plotted in Fig. 4, In Fig. 4 the size of the circles is proportional to the $\mathrm{Cu}$ $3 d_{x^{2}-y^{2}, \downarrow}$ character of the respective states. The comparison shows a substantial $\mathrm{Cu} 3 d_{x^{2}-y^{2}, \downarrow}$ contribution to the 


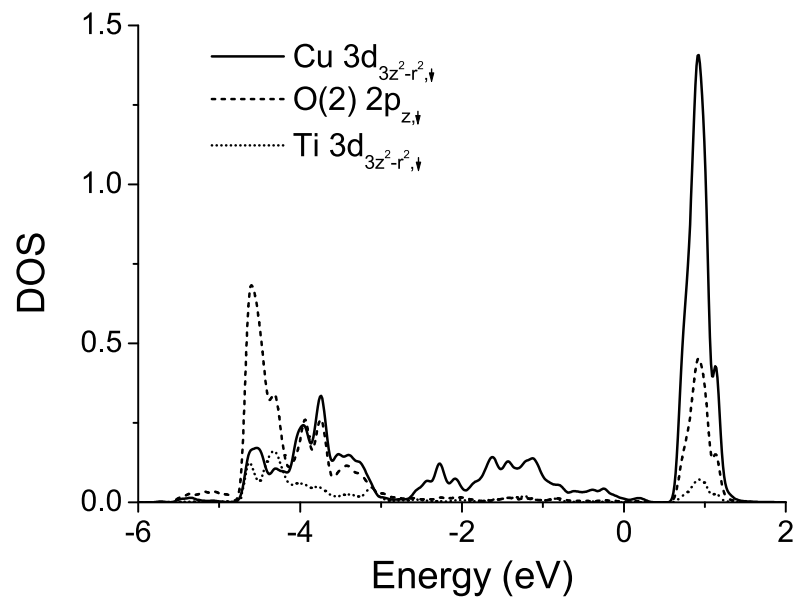

FIG. 6: Projected $\mathrm{Cu} 3 d_{3 z^{2}-r^{2}, \downarrow}, \mathrm{O}(2) 2 p_{z, \downarrow}$ and $\mathrm{Ti}$ $3 d_{3 z^{2}-r^{2}, \downarrow}$ orbital densities of states at the interface between $\mathrm{CuO}_{2}$ and $\mathrm{SrTiO}_{3}$ in the relaxed heterostructure (LSDA+U studies). The zero of energy is at the Fermi level.

$2 p$ states of oxygens in the $\mathrm{CuO}_{2}$ planes which are located below the Fermi level. This contribution is especially significant at the energies about $-(0.6-0.8) \mathrm{eV}$, at $-2.5 \mathrm{eV}$ as well as at about $-(3.8-4) \mathrm{eV}$. Specifically, the integration of the partial densities of states below the Fermi level shows an increase of the population $\Delta n_{x^{2}-y^{2}, \downarrow}$ of $\mathrm{Cu} 3 d_{x^{2}-y^{2}, \downarrow}$ levels by about 0.2 electrons in the relaxed structure. To check the numerical accuracy of this result we have also calculated the increase of the electron concentration from equation (6) as $\Delta n_{x^{2}-y^{2}, \downarrow}=n_{x^{2}-y^{2}, \downarrow}^{0}-$ $n_{x^{2}-y^{2}, \downarrow}^{1}=\left(\varepsilon_{x^{2}-y^{2}, \downarrow}^{1}-\varepsilon_{x^{2}-y^{2}, \downarrow}^{0}-\Delta \varepsilon_{x^{2}-y^{2}, \downarrow}^{10(0)}\right) / U_{\text {eff }}=0.25$ with $\Delta \varepsilon_{x^{2}-y^{2}, \downarrow}^{10(0)}=\varepsilon_{x^{2}-y^{2}, \downarrow}^{1(0)}-\varepsilon_{x^{2}-y^{2}, \downarrow}^{0(0)}$, which agrees well with the value obtained by the direct integration. Besides the $3 d_{x^{2}-y^{2}, \downarrow}$ state, the relaxation also results in an increase of the population of $3 d_{x^{2}-y^{2}, \uparrow}$ orbital by about 0.1 electrons, as compared to the system with $h=2.2 \AA$.

Using the values of the band centers given in Table \. we find from Eq. (5) that the minimal energy shift $\Delta \varepsilon_{3 z^{2}-r^{2}}^{10}$ sufficient for orbital reconstruction is given by $\Delta \varepsilon_{3 z^{2}-r^{2}}^{10} \approx 1 \mathrm{eV}$. As the typical values of the crystal fields expected in this type of systems can approach $2-3 \mathrm{eV}$, we can conclude that the mechanism of the orbital reconstruction realized through the stabilization of the extended $\mathrm{Cu} 3 d^{8}$ configuration shown in Fig. 2 (bottom panel) appears to be a plausible scenario. We note that this reconstruction occurs despite a strong local Coulomb repulsion $U=8 \mathrm{eV}$ which is overcome by the modifications of the structure of the $\mathrm{Cu} 3 d_{x^{2}-y^{2}, \downarrow}$ and $3 d_{3 z^{2}-r^{2}, \downarrow}$ bands.

\section{ELECTRONIC DENSITY AT ORBITAL RECONSTRUCTION}

To understand better the nature of the reconstruction of $\mathrm{Cu} 3 d_{x^{2}-y^{2}, \downarrow}$, we also analyze the $(x, z)$ electron density maps (represented in Fig. 5) in the energy range of the bonding states $\mathrm{Cu} 3 d_{x^{2}-y^{2}-\mathrm{O}} 2 p$. Here the $z$-axis is directed perpendicular to (001). The decrease of the interface distance $h$ leads to stronger electron repulsion in the nearest $\mathrm{CuO}_{2}$ and $\mathrm{SrO}$ layers. The effect of this repulsion is clearly visible in the bottom panel of Fig. 5 ( $h=1.83 \AA)$ which shows electron depletion in the spatial region between $\mathrm{Cu}$ (located at $x=2 \AA, z=4.5 \AA$ ) and oxygen $\mathrm{O}(2)(z=2.5 \AA)$ in the SrO-plane. The decrease of the electron density along the $z$-direction has an extended character and involves $\mathrm{Cu} 3 d_{3 z^{2}-r^{2}, \downarrow}, \mathrm{O}(2)$ $2 p_{z, \downarrow}$ and $\mathrm{Ti} 3 d_{3 z^{2}-r^{2}, \downarrow}$ orbitals which may be interpreted as reduced hybridization between these orbitals. In Fig. 6 this decrease is manifested through a peak centered at $E=1 \mathrm{eV}$ above the Fermi level which appears due to the splitting of the $3 d_{3 z^{2}-r^{2}}$ bands. The peak remains significant even in the density of Ti $3 d_{3 z^{2}-r^{2}, \downarrow}$ states which are more distant from the interface. The consequent increase of the difference between the electron occupancy of the majority and minority spin states also leads to the enhancement of the magnetic moment of $\mathrm{Cu}$ from $0.65 \mu_{B}$ to $0.86 \mu_{B}$, an effect discussed in the previous section.

In contrast to the electron depletion in $z$ direction, in the $\mathrm{CuO}_{2}$-plane the interface relaxation results in a strong charge hybridization between $\mathrm{Cu}$ and $\mathrm{O}(1)$ which is presented in more detail in Fig. 7 To estimate the modification of electron charge resulting from the combination of (i) electron depletion across the interface and (ii) electron accumulation in $\mathrm{CuO}_{2}$ planes, we have calculated planar $(x, y)$ and orthogonal $(z)$ contributions to the electron density. To obtain the orthogonal part (i) (electron depletion), we have extracted the charge density in the range between $0.5 \mathrm{eV}$ and $1.4 \mathrm{eV}$ which corresponds to the new DOS-peak. The direct integration of the obtained spatial density profiles results in the value $n_{e}^{z} \approx 1.45$ carriers per unit cell. This value should be corrected by $\Delta n_{e}^{z}$ on account of an overlap with the states $3 d_{x^{2}-y^{2}}, 2 p_{x}$ and $2 p_{y}$ which are shifted towards lower energies in the relaxed system (see Fig. 31). The integration of the corresponding projected DOS parts gives $\Delta n_{e}^{z}=0.28$. It should be noted that the latter integration has been performed without consideration of extra charge in the interstitial regions which should give additional contributions to $\Delta n_{e}^{z}$. In our analysis, the interstitial contributions are expected to be small and the resulting $\Delta n_{e}^{z}$ does not exceed the maximal value of about 0.45-0.5. As a consequence, the obtained modification of the depleted charge density $\Delta n_{i t f}=n_{e}^{z}-\Delta n_{e}^{z} \approx 1$. This implies that approximately one electron per unit cell is depleted from the $3 d_{3 z^{2}-r^{2}}$ and $2 p_{z}$ orbitals of the interfacial bonding complex $\mathrm{Cu}-\mathrm{O}(2)-\mathrm{Ti}$. In the interfacial stack $\mathrm{CuO}_{2}-\mathrm{SrO}-\mathrm{TiO}_{2}$, about $70 \%$ of the depleted charge is located in the $\mathrm{CuO}_{2}$ layers which suggests a strong spa- 


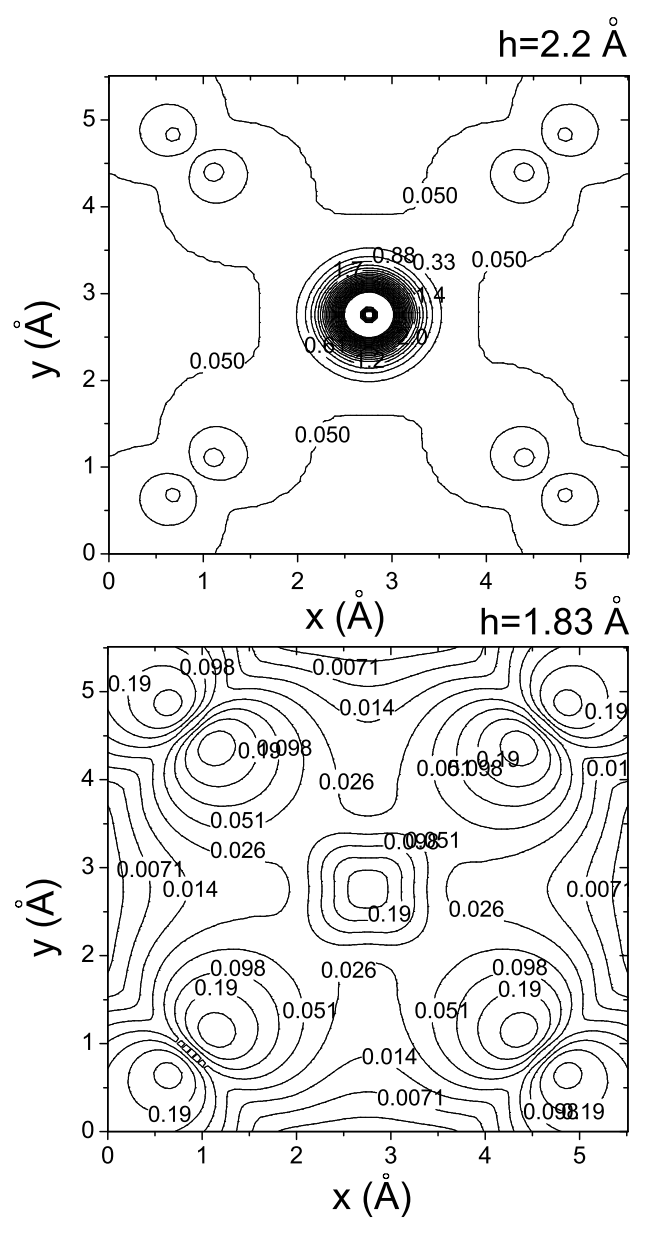

FIG. 7: Electron density map (in e/ $\AA^{3}$ ) in the energy range $-4.5 \mathrm{eV} \leq E \leq-2.5 \mathrm{eV}$ below the Fermi level in $(x, y)$ plane for unrelaxed and optimized interface distances. Here $z / c=0.7$.

tial charge confinement to the $3 d_{3 z^{2}-r^{2}}$ orbitals of $\mathrm{Cu}$.

To calculate the amount of the electrons accumulated in the $\mathrm{CuO}_{2}$ planes of the relaxed structure (contribution (ii)), we performed the integration of the hole charge from the Fermi level to the top of the $\mathrm{O}(1)$ valence band which gives $n_{h}^{x y}=1$ holes. These holes are located in the $2 p_{x, y}$ states of $\mathrm{O}(1)$ hybridized with the $3 d_{x^{2}-y^{2}}$ orbitals of $\mathrm{Cu}$. The comparison with the value $n_{h}^{x y}\left(h_{1}\right)=2$ for the unrelaxed system provides the resultant amount $n_{e}^{x y}=1$ for the additional electron charge accumulated due to structural relaxation. It is remarkable that in their combination, both contributions (i) and (ii) give the total positive charge $+2 \mathrm{e}$ required for the overall neutrality of the relaxed system.
Therefore, in this mechanism for the orbital reconstruction one can distinguish two key factors which are responsible for the stabilization of the extended $\mathrm{Cu} 3 d^{8}$ configuration near structurally relaxed $\mathrm{CuO}_{2} / \mathrm{SrTiO}_{3}$ interfaces: (i) the depletion of the electrons from a $3 d_{3 z^{2}-r^{2}}$ orbital of $\mathrm{Cu}$ hybridized with $2 p \mathrm{O}(2)$ and $3 d_{3 z^{2}-r^{2}}$ states of $\mathrm{Ti}$, a process caused by the splitting of the $3 d_{3 z^{2}-r^{2}}$ bands; (ii) the increase of electron charge density in the $\mathrm{Cu} 3 d_{x^{2}-y^{2}, \downarrow}$ orbitals which are strongly hybridized with the $2 p_{x, y}$ bands of the oxygens $\mathrm{O}(1)$ in the $\mathrm{CuO}_{2}$ planes. This increase is accompanied by a shift of the $3 d_{x^{2}-y^{2}, \downarrow}$ band towards lower energies and it provides the energy gain sufficient for the orbital reconstruction. It is worth noting that the second stage (increase of electron concentration on $\mathrm{O}(1) 2 p$ and $\left.\mathrm{Cu} 3 d_{x^{2}-y^{2}, \downarrow}\right)$ in fact counterbalances the electron depopulation in stage (i). In this way, it minimizes the lost of energy due to the splitting of $\mathrm{Cu} 3 d_{3 z^{2}-r^{2}}$ bands with the orbital reconstruction. The obtained charge self-regulation is in agreement with the negative feedback mechanism discussed in Ref. 13 which supports its general character for the transition metal oxides.

\section{CONCLUSIONS}

To summarize, we note that in the orbital reconstruction mechanism, the effective change of the valency of $\mathrm{Cu}$ due to the splitting of $3 d_{3 z^{2}-r^{2}}$ bands appears to be a plausible and realistic property. For the cuprate-titanate interfaces, the resulting electronic state is characterized by the electronic configuration $3 d^{5 \uparrow, 3 \downarrow}$ of $\mathrm{Cu} 3 d$ orbitals extended by the changes of orbital occupancies of ions in the neighbouring $\mathrm{SrO}$ and $\mathrm{TiO}_{2}$ planes. Surprisingly, the strong hybridization between $\mathrm{O} 2 p_{x, y}$ and $\mathrm{Cu} 3 d_{x^{2}-y^{2}, \downarrow}$ states of $\mathrm{CuO}_{2}$ planes plays an essential role, making even moderate crystal fields acting on $\mathrm{Cu} 3 d_{3 z^{2}-r^{2}}$ orbitals sufficient for the orbital reconstruction at $\mathrm{CuO}_{2}-\mathrm{SrO}$ interfaces and the consequential splitting of the $3 d_{3 z^{2}-r^{2}}$ states through the onsite Coulomb interaction $U$. An increase of the magnetic moment of $\mathrm{Cu}$ (from $0.65 \mu_{B}$ to $0.86 \mu_{B}$ ) is predicted for the reconstructed interface state.

\section{ACKNOWLEDGEMENTS}

This work was partially supported through the DFG SFB-484. The author thanks Thilo Kopp for helpful discussions. Grants of computer time from the LeibnizRechenzentrum München are gratefully acknowledged.
1 A. Ohtomo and H.Y. Hwang, Nature 427, 423 (2004); Nature 441, 120 (2006).

2 S. Thiel, G. Hammerl, A. Schmehl, C.W. Schneider, and
J. Mannhart, Science 313, 1942 (2006).

3 A.D. Caviglia, S. Gariglio, N. Reyren, D. Jaccard, M. Gabay, S. Thiel, G. Hammerl, J. Mannhart, and J.- 
M. Triscone, Nature 456, 624 (2008); N. Reyren et al., Science 317, 1196 (2007).

4 This mechanism was demonstrated for a polar surface of $\mathrm{K}_{3} \mathrm{C}_{60}$ by R. Hesper, L.H. Tjeng, A. Heeres, and G.A. Sawatzky, Phys. Rev. B 62, 16046 (2000).

${ }^{5}$ C. Noguera, F. Finocchi, and J. Goniakowski, J. Phys.: Cond. Matt. 16, S2509 (2004).

6 J. Chakhalian et al., Science 318, 1115 (2007).

7 F.C. Zhang and T.M. Rice, Phys. Rev. B 37, 3759 (1988).

8 M. van Veenendaal, Phys. Rev. B 78, 165415 (2008).

9 N. Pavlenko, E. Elfimov, T. Kopp, and G.A. Sawatzky, Phys. Rev. B 75, 140512(2007).

10 N. Pavlenko and T. Kopp, Electronic charge and orbital reconstruction at cuprate-titanate interfaces, in
High-Performance Computing in Science and Engineering (Springer, 2008), pp. 697-707.

11 R.E. Watson, H. Ehrenreich, and L. Hodges, Phys. Rev. Lett. 24, 829 (1970).

12 V. Eyert, U. Schwingenschlögl, and U. Eckern, Europhys. Lett. 70, 782 (2005).

13 H. Raebiger, S. Lany and A. Zunger, Nature 453, 763 (2008).

14 P. Blaha et al., WIEN2K, An Augmented Plane Wave + Local Orbitals Program for Calculating Crystal Properties, ISBN 3-9501031-1-2 (TU Wien, Austria, 2001).

15 V.I. Anisimov et al., Phys. Rev. B 48, 16929 (1993). 